\title{
Developing Web Content Management Systems - from the Past to the Future
}

\author{
Andrei Yermolenko, ${ }^{1, *}$ and Yuriy Golchevskiy ${ }^{1,2}$ \\ ${ }^{1}$ Sorokin Syktyvkar State University, Institute of Exact Sciences and Information Technologies, \\ Oktyabrskiy Ave., 55, Syktyvkar, Russian Federation \\ ${ }^{2}$ Syktyvkar Forest Institute, Lenina St., 39, Syktyvkar, Russian Federation
}

\begin{abstract}
The article presents an overview of the main stages of development and formation of content management systems as a component of software, in conjunction with the material base. The need of a society in creation and development of systems is revealed. The main elements of the mentioned systems, their functionality, and user groups are described. The features of modern content management systems are presented. The change in approaches to the development of such systems from monolithic to distributed systems is outlined. Further formation of Web-content management systems is seen in the development of systems with open source code, using Web 3.0, refining tools analysis of the quality of content, support for business B2B and B2C, interface development, active use of headless CMS, support of a new level of SEO, implementation of artificial intelligence algorithms.

Keywords: content management systems
\end{abstract}

\section{Introduction}

Modern websites provide companies with the opportunity to promote their products and services worldwide, from anywhere at any time, 24 hours a day, increasing the number of potential customers. One of the factors behind the significant growth of websites is the emergence and development of Web Content Management Systems (WCMS) [1]. WCMS is a narrower concept than CMS - content management system. In some countries, including Russia, the term CMS is used among web-masters instead of WCMS. The main advantage of WCMS is the separation of the site designer from its information content. Thus, site designer turns into a simple tool, which does not require possess the skills of HTML-page layout and programming. This allows a sufficiently large number of people who do not have skills in programming, layout and web design, easy to develop and operate a web resource. The mechanisms and features provided by modern WCMSs satisfy a wide range of needs and requirements of small and medium-sized businesses due to the high customizability and, at the same time, the low cost of development, implementation and maintenance.

\footnotetext{
* Corresponding author: ea74@list.ru
} 


\subsection{Related works}

There are quite a lot of materials on the Internet devoted to the use of different WCMSs. A significant number of such materials are oriented towards developers of web-resources based on specific WCMSs, which is caused by the desire of the creators to popularise and promote their systems. This allows to be guided in actual functionality, tools in use, and further development. When preparing this article we studied descriptions of web-content management systems on the official sites of developers and communities of free systems WordPress, Drupal, Joomla, ucoz, MODX, phpbb, fluxbb and proprietary systems 1CBITRIX, Contentstack, Wix, Mogut.CMS, UMI.CMS, and Netcat. We have also studied various professional and educational standards such as Professional standard 06.035 "Web and multimedia developer", Worldskills Russia (7WSI "Web Design and Development") and others, and analyzed the relationship between educational and professional standards, for example, the study cited in [2].

A change in the subject matter of WCMS articles can be noted. While initially articles focused on the purpose [3, 4], difficulties of implementation and doubts of using such systems $[5,6]$, over time the focus has shifted to demonstrating successful experience in certain subject areas [7], creating new systems [8,9] and justifying WCMS skills in the digital economy [10].

\subsection{Purpose and objectives}

We have been participating and teaching in educational programmes related to web technologies and the use of WCMS for 20 years. We have participated in the development of various web projects using WCMS, e.g.:

- "Komi Republic in numbers".

- Journal site "Bulletin of Syktyvkar University. Series 1: Mathematics. Mechanics. Informatics" and "Human. Culture. Education".

- Creation of library web resources.

The experience gained allows us to describe the advantages and disadvantages of modern content management systems, and to trace the development of such systems practically from their inception to the present day.

The purpose of this research is to review how the development of computer hardware, technology and general requirements for information systems has affected the development of WCMSs, and to reflect on the future of content management systems based on current industry trends.

\section{Methods}

This study is based on the use of theoretical and empirical research methods. The theoretical method allowed analysing the development of web content management systems, to analyse the dynamics of transformation of the requirements of modern WCMS. An analysis of publications and scientific discussions on the subject was carried out.

The empirical method was applied in the process of observation and comparison of data obtained over many years of the authors' practice in the use of WCMS, interviewing representatives of professional communities for a deeper understanding of the described problem. 


\section{Results and Discussion}

\subsection{Background to WCMS}

The Internet gained widespread popularity with the advent of the World Wide Web in 1991. Initially it was believed that the beauty of websites was not important, the main thing was information. It was concerned with weak, by today's standards, computer hardware i80486 processors with clock frequency of $16 \mathrm{MHz}, 32 \mathrm{Mb}$ RAM and data transfer rate of 19.2 Kbit/s. Under these conditions, speed of transfer of simple page in size of $200 \mathrm{~Kb}$ containing text and simple picture was more than one and a half minute.

The search of information in Internet was not different from the search of information in the library - it was necessary to know the exact address of the site or to use paper publications, like the yellow pages of Internet.

This caused low activity of companies in the Internet. However, with the advent in 1994 of the first thematic server Yahoo and the first search server WebCrawler search for information greatly simplified, business has received new tools for development and companies have become more interested in disseminating their information network Internet.

It should be noted that the first web sites were static web pages created manually based on simple text HTML-files. The creation of web pages at that time was a non-trivial task, because the content of the site was closely intertwined with programming. Therefore, it was possible for people with special skills in layout and programming to create websites.

As the power of personal computers increased - the Intel Pentium processor with a clock frequency of $133 \mathrm{MHz}$ was released in 1995 - the Internet became visual and functional. The JavaScript programming language (1995) and cascading style sheets CSS (1996) appeared. Various standards began to emerge, which would later be referred to as the "browser war". In December 1997, HTML 4.0, also called Dynamyc HTML, was released, bringing web pages somewhat "alive". The use of the Document Object Model (DOM) for HTML and XML documents makes them more interactive.

In parallel with the client side, server-side programming was actively developed in the 90s. Programming languages appear, such as PHP (1995), Java (1995), Active Server Pages technology (1996), MySQL database management system (1994).

In 1996, a full-fledged scripting language called CFML began to be actively used. Data input processing using Common Gateway Interface and the programming languages Perl and Python became the norm. From 1995 to 1997, the use of server-side scripting became very popular. However, the introduction of PHP and ASP began to actively compete with server-side scripting in use when generating content, sent from the server to the web browser. In 1999 Java Server Pages (JSP), built on Java programming language, started to be introduced and quickly became quite popular among web developers.

In 2005 AJAX technology appeared, which allowed to request and receive data for updating web pages without reloading, which was considered to be very progressive.

Rapid development of software helped divide the site into functional blocks - content (MySQL, HTML), design (CSS) and business logic (PHP, JavaScript).

\subsection{The first CMS}

The technologies described above led to an exponential increase in the volume of information placed on the Internet in the late 90s. Businesses had the desire and opportunity to use corporate websites [11] in their activities. However, the support of web-sites was carried out mainly manually by programmers. This made it somewhat difficult to publish 
corporate web content in a timely manner. Thus the need arose for systems to automate and optimize the process of working with content, that is, content management systems.

The first CMSs began to appear in the mid-1990s. They were developed by organizations and companies independently, using existing technology, and were targeted primarily at the needs of those specific organizations.

From 1995 to 1997 enterprise content management systems like FileNet, StoryBuilder, Interwoven, Documentum, FatWire, FutureTense and Inso appeared.

In addition we can mention the success of GeoCities, created in 1994, the world first massively free hosting service and, in fact, the prototype of modern social networks. GeoCities can in all likelihood be called the first mass WCMS to allow users to manage their own websites.

\subsection{Formation of the WCMS}

Since the early 2000s, there has been an active creation of Internet content management systems in a professional and large-scale manner. The term WCMS - Web Content Management System - emerges, emphasising that CMS is a broader term to include content management for intranets, archives and business operations.

At this time, the position was clearly established that a modern website should conventionally consist of two parts - the designer and the content. The designer should be handled by programmers, and the filling of the site with information by professionals in the subject area relevant to the subject of the content. This finally allowed connecting to the creation of sites a large number of people, which led to a further increase in the quantity and quality of information on the Internet.

The development of CMS was also influenced by a significant improvement of the material base - computers to the middle of zero years were completed with processors with a clock frequency of $2 \mathrm{GHz}, \mathrm{RAM}$ up to $4 \mathrm{~Gb}$, data transfer rate in the Internet up to 50 $\mathrm{Kb} / \mathrm{sec}[12,13]$. Such characteristics of computers allowed creating and "consuming" colorful and dynamic sites. Great attention was paid to the sites convenience, as it became one of the criteria influencing the attendance.

Open-source CMSs such as Mambo (2000), Drupal (2001), WordPress (2003) and Joomla (2005) appeared during this period. Commercial CMSs such as NetCat (1999), Shop-Script (2002), Bitrix: Site Management 3.0 (2003), CS-Cart (2004).

This development has allowed forming the following four main functions of WCMS:

1. Creation - providing the authors of convenient and familiar means of creating content in the form of WYSIWYG editors.

2. Management - storing content in a single repository. Includes storage, version tracking, access control, integration with other information systems and document flow management.

3. Publishing - automatic placement of content to the user's terminal. Appropriate tools automatically adapt the appearance of the page to the design of the entire site.

4. Presentation - additional functions to improve the form of data presentation; for example, it is possible to build navigation on the repository structure.

A typical WCMS using a database is shown in Figure 1.

Almost immediately, the following user groups were formulated:

- Users - the main consumers of content.

- Designers - template developers.

- Administrators for user management.

- Implementation team - installation and configuration of the system.

- Authors (content makers) - content creators. 
The uniqueness of sites created with WCMS is achieved through a large number of both paid and free templates, plug-ins and access to CSS. by:

Disadvantages of the first mass-produced WCMS that can be highlighted as the years go

- CSS configuration is not visualised, knowledge of CSS commands is required.

- Limited functionality of WYSIWYG editors, some functions were implemented only through HTML tags, e.g. working with tables.

- Limited number of people allowed generating content.

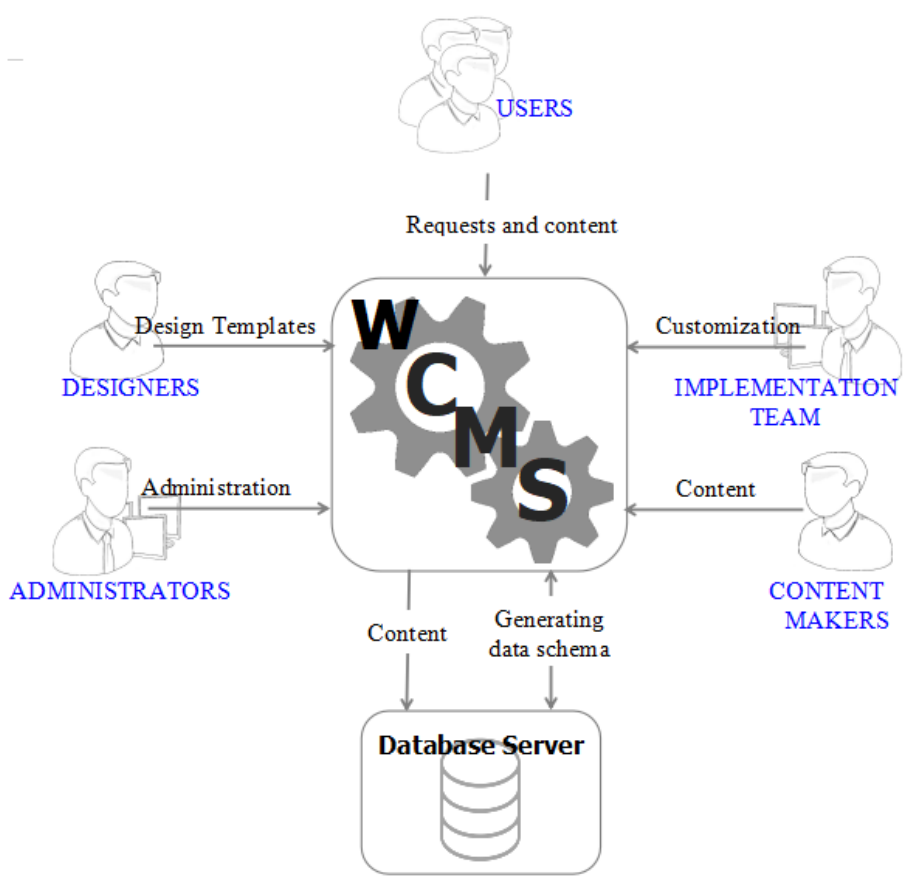

Fig. 1. Functioning diagram of a typical WCMS

\subsection{Modern WCMS}

Several important factors have had a decisive influence on the development and presentation of modern WCMSs.

Firstly, it is the further development of computer hardware - the computing power of a modern smartphone exceeds that of a personal computer of 20 years ago. Characteristics of modern budget PC: 4-6 cores, clock frequency $-3 \mathrm{GHz}, 8 \mathrm{~GB}$ of RAM, speed of data transfer to Internet - 100 Mbit.

The second is the emergence of the Web 2.0 concept and technology [14] in 2005, social networking, cloud computing.

Web 2.0 has opened new horizons for the Internet in general and for WCMS in particular. Not just a few individuals, but whole communities are now contributing to the information sites, and this has led to a considerable increase in the amount of information available. At the same time, there was a need for significantly simplified tools for working with content - wiki markup appeared and online editors for many popular applications were created. This has led to a significant demand for interfaces aimed at non-professionals in information systems - new more convenient and functional versions of WCSS WYSIWYG editors are appearing, and the installation and initial setup of WCMS takes minutes. 
The rapid growth of social networks has led to the need to integrate WCMS with social networks. Plug-ins are appearing that allow you to automatically link to websites on social networks, registration via social networks appears.

The third significant factor is the rapid development of mobile technology, the increase in traffic from mobile devices and its noticeable influence on trends in building websites and delivering content to the consumer. The emergence of the iPhone and Android smartphones in 2007 - 2008 and smart tablets in 2010 has led to a dramatic increase in mobile Internet traffic, and in 2014 it exceeded the traffic of desktop solutions in the US (this is now a global trend). This led to difficulties in delivering content correctly to consumers on different devices, leading to different content delivery channels (for desktop solutions and mobile devices), and further to a paradigm shift in WCMS development. At first, it obliged WCMSs to create adaptive website designs that look the same on different devices, and then to realize that it was necessary to develop systems with omnichannel content delivery ready to support all existing and emerging channels.

The development of technology, on the one hand, leads to technological sophistication of websites - virtual reality begins to be supported, websites become more interactive, contain a large amount of multimedia content and tools for its online processing, and, on the other hand, to a simplification of design or a change in its paradigm (for example, with an orientation towards mobile devices), and the development of social orientation of the Internet strongly influences trends in content creation. The main approach today is Content First, a focus on content and the blurring of the line between user and interface. There are no special restrictions, the main thing is to create the right atmosphere for the user, to create controls, content, orienting on the person that is natural language and familiar forms [15]. The modern Internet is not just digital pages but an environment in which a modern person wants to exist organically, which requires additional tools for WCMS as well.

A great variety of sites leads to the development of search engines, which have to introduce new criteria for sorting sites in search results. There appear such numerical characteristics as PR [16], Alexa Rank [17], TIC and IKS indicators [18]. SEO (Search Engine Optimization) and its support by additional plug-ins appears consequently.

The transfer of large volumes of information brings the problems of information security to a higher level. Information in many countries around the world is now carefully protected not only technically, but also by law.

WCMS features such as URL processing, RSS feeds, visitor comments, chatbots, version tracking systems, permission systems, drag-and-drop, templates, integration with ecommerce tools, analytics tools, integration with ECM and ERP systems, various platforms widely used by business, etc. are also developing.

Much attention is being paid to studying buyer behaviour in B2B and B2C systems, introducing new approaches to increase sales [19], and separate websites are appearing to support CRM, as well as modules for implementation in content management systems.

The emergence of new technologies entails a change in approaches to web resource development. Along with traditional front-end and back-end development, microservices architecture is emerging [20].

The emergence of cloud computing in 2006 allowed to look at the placement of sites on the Internet from a new perspective - WCMS services began to be provided as a service (the concept of Software as a Service), so one of the objective trends of the modern world was a significant increase in the number of existing Internet sites. In 2016, the number of websites passed the 1 billion mark. The number of websites as of January 2021 was over 1.8 billion and the number of domain names exceeded 370 million [21].

There are now various ways of presenting information - computer, smartphone, tablet, smart watch, Internet of Things, as well as digital signage, widgets, microservices and even features on websites and new ones are constantly emerging. It is becoming an unacceptable 
luxury to create an app for every device. The idea of actively using MVC technology to separate the application data, user interface and control logic into three separate components has emerged. As a result, so-called headless CMSs are being developed with common structured content, but different interfaces implemented using different architectural features. Potential advantages of headless CMS include:

- No interface system and the integration of content management tools through APIs, which allows content to be published on any device or channel, as there is effectively a separation of formatting from content.

- Belonging of headless CMS to the category "content as a service", which extends to any third-party applications integrated into the system, which increases the possibility of integration and increases the security of the resource in the case of a reliable provider of such services.

- Good scalability provided by a cloud-based solution that provides the flexibility to customise the entire infrastructure depending on the need.

\subsection{Directions for WCMS development}

In the light of this history, let us formulate the directions of WCMS development as seen by the authors.

1. Leading free and open-source WCMSs in the coming years.

2. Growing interest to use elements of Web 3.0, e.g. decentralised Internet infrastructure, blockchain, machine learning, semantic web and Internet of things, using some approaches inherent to semantic programming.

3. Development and increased adoption of content quality analysis tools in the CMS, which will enable the creation of better and/or more personalised content that includes corporate content and analytics.

4. Development and more active implementation of business analysis and promotion tools in the CMS. Already, analysis of consumer activity on the site, sales funnels, etc., is almost a standard offering for the webpage builders. Such analysis tools will be offered more actively.

5. Development of interfaces - interfaces that are as simple and intuitive as possible are needed. Development of voice and 3D interfaces, interface personalisation is expected.

6. Development of headless CMS, separating the data layer from the display layer and allowing more rational use of applications for different devices.

7. SEO support comes to the fore - today it is no longer enough just to make a website, it is necessary to spend effort on its promotion. Therefore, further development of SEO plugins is demanded, and it is necessary to include an SEO specialist in the WCMS workflow (Fig. 1).

8. Implementation of artificial intelligence algorithms to manage and provide corporate content and personalise content for the end user, analyse the usefulness and quality of corporate content and analyse the behaviour of web resource users.

\section{Conclusions}

In conclusion, the development of WCMS is directly related to the development of technology, hardware and software.

The use of WCMS allows non-specialists in the field of information systems to easily make a beautiful and functional website. It is enough to create several websites using two or three systems to master modern technologies of website creation (we emphasize that we 
speak only about website creation without taking into account its aesthetic, businessoriented features and various functional orientations).

\section{References}

1. P. Cigoj, B.J. Blazic, IEEE, 7, 175466-175473. (2019) DOI: $10.1109 /$ ACCESS.2019.2957573.

2. N. Nasyrov, N. Gorlushkina, A. Uzharinskiy, Digital Transformation and Global Society. 1038, 565 - 573. (2019) DOI: 10.1007/978-3-030-37858-5_48.

3. B. Caron, D. Toole, P. Wicks, S. Miller, Earth Science Informatics. 4. 191-196. (DEC 2011) DOI: $10.1007 / \mathrm{s} 12145-011-0090-7$.

4. V.G. Ibarra, H.M. Gomis, D.P. Caparros, J.A.P. Benito, ICE-B 2007: Proceedings of the Second International Conference on E-Business. 183. (2007).

5. C.R. Lanier, Technical Communication. 59. 99-111. (MAY 2012).

6. D. Goans, G. Leach, T.M. Vogel, Library Hi Tech. 24. 29-53. (2006) DOI: $10.1108 / 07378830610652095$.

7. L. Vogt, R. Baum, P. Bhatty, C. Kohler, S. Meid, B. Quast, P. Grob, The Journal of Biological Databases and Curation. baz067. (2019) DOI: 10.1093/database/baz067.

8. V. Vysotska, A. Demchuk, V. Lytvyn, Radio Electronics Computer Science Control. 4. 121-135 (2019) DOI: 10.15588/1607-3274-2019-4-12.

9. P. Cigoj, B.J. Blazic, IEEE Access. 7. 175466-175473. (2019) DOI: 10.1109/ACCESS.2019.2957573.

10. Yu. Golchevskiy, E. Novokshonova, A. Yermolenko, Advances in Economics, Business and Management Research, Atlantis Press. 156, 291-296. (2020) DOI: 10.2991/aebmr.k.201205.048.

11. B. Moller, C. Brezing, D. Unz, Behaviour \& Information Technology. 31. 739-751. (2012) DOI: 10.1080/0144929X.2011.642893.

12. PC MAGAZINE / RUSSIAN EDITION , 1. 116-122. (2005)

13. 2005. F.A.Q. for choosing a modem https://forum.ixbt.com/topic.cgi?id=32:11958 (accessed 20 March 2021).

14. T. O'Reilly, What Is Web $2.0 \mathrm{https} / /$ www.oreilly.com/pub/a/web2/archive/whatis web-20.html?page=1 (accessed 20 March 2021).

15. Yu.V. Golchevskiy, Economics. Information technologies. 47 (2). $432-440$ (2020) DOI: 10.18413/2687-0932-2020-47-2-432-440.

16. A. Dixit, V.S. Rathore, A. Sehgal, Advances in Intelligent Systems and Computing. 841. 535-540. DOI: 10.1007/978-981-13-2285-3_63.

17. I. Najafi, M. Kamyar, A. Kamyar, Tahmassebpour M. IEEE ACCESS. 5. 1228612292. DOI: 10.1109/ACCESS.2017.2720118.

18. What is a site quality index (ICS)? (in Russian) Available at: https://yandex.ru/support/webmaster/site-quality-index.html (accessed 20 March 2021).

19. N. Huang, T.S. Sun, P.Y. Chen, J.M. Golden, Information Systems Research. 30. 805818. (2019) DOI: 10.1287/isre.2018.0832.

20. Yu.V. Golchevskiy, A.V. Yermolenko, Bulletin of Syktyvkar University. Series 1: Mathematics. Mechanics. Informatics.(35) 2. 25 - 36 (2020).

21. Internet Stats \& Facts (2021). Available at: https://websitesetup.org/news/internetfacts-stats/ (accessed 20 March 2021). 